\title{
Relationship Between Entrepreneurshipand Employability Among Nigerian Tertiary Institution Graduates
}

\author{
${ }^{1}$ Oladipo kolaposakiru, ${ }^{2}$ Gafar Olanrewaju yusuf ${ }^{1}$ Mohammed Abdullahi, \\ ${ }^{1}$ Aliyu yero, ${ }^{1}$ Narges Kia. \\ ${ }^{I}$ Department of Human Resource Development, Universiti Putra Malaysia \\ ${ }^{2}$ Faculty of Management and Human Resource Development, Universiti Technnology Malaysia.
}

\begin{abstract}
Many graduatesin Nigeria areunemployed, regardless oftheirqualifications, they're employable yetthey aren'temployed. The lack ofabilityfrom thegreatereducational facilitiesto meetthe requirements ofthosegraduatesin addition topromotionof monetary selfrelianceandself sufficiency hasresultedinto youths joblessness (unemployment). Thesehaveput intorise in restiveness among Nigerian youths. Thestudy looks into the relationship between entrepreneurship and employability among Nigerian graduates. Thepaper, talked aboutthe idea ofentrepreneurshipand employability. Since employability is theactionof gettingqualificationthat allowsanyone toemployed, the studyalsochecked outthe fundamentalabilitieswhich will makeone employable andsimultaneouslybecomingselfsufficient.Alsotalked methodsforwardinmarketingentrepreneurshipamong howteachingofcreativenessandproblemfixingabilitiesmight help reposition Nigerian graduates.

aboutare

Nigerian youths and just
\end{abstract}

Key word : employability, entrepreneurship, Nigerian graduates, youth.

\section{Introduction}

Nigeria similar tounder developednations around the worldis faced withplenty ofproblemsandharshdetailsincludingpoverty, unemployment, conflictsandailments. Thesesituationsposegreatchallengesfor the entirelife ofindividualsin lots ofunder developednationsthereforewithexercisingofeducatedmalesand girlswho is able tofunctioneffectivelyinside thesocietythrough whichtheylive in. There arelargeunemploymentof Nigerian schoolsgraduateswithin the country, this problemismentionedbeing traceable for the disequilibrium betweenworkmarketneedsandinadequateessential employable capabilitieswith thegraduates, (1). Thisapparentcriticalskillgapsinhibitthe development of youths as well as theentiregrowth and development ofthecountry. The earthisfacinga veryhard timeand Nigeria just like anationcannotrun awayout of this, ratherwe areto providetechniques tosomecouple of thoseproblemsfacingus. A coupleof themajorproblemsfacing Nigeria todayjust like adevelopingcountryincludeunemployment, poverty, poorhealth careservices, youth restiveness, insecurity, etc. Nigeria cannoteasilymakeheadway, once weneglectorhaven'tdevelopedease ofentrepreneurshipwhich assists the employability within our youths. Anidlesoulcan be adevilsworkshop. Thispaperaddressesthemassgraduateunemploymentin Nigeria, along with thefailureofgraduatesto takeadvantage of tacit andexplicitunderstandingacquiredutilizing theirstudiesin an organizational way.

Entrepreneurship has a several potential benefits in theobjectivesofreducingunemploymentamong Nigerian graduates, since itplaysan important roleindevelopmentand growth oftheeconomy. It assists marketinnovation whichinspiresgraduatestodiscovernewtechniquesfordoingthingsthroughexperiencebasedlearnin g. However, the Nigerian youths mentioned are being confronted withpoverty, unemployment, urbanization, inadequatecapacityandcapabilitiesneeded tograduallyslowlymove theeconomyforward. Povertythat's apressurefor Helps/Helps is very common. For the reason thattheyouthfaces unemployment andinadequatenecessaryproductivecapabilitiesto keepbodyandsoultogether. This reality leavesall of them withnosignificantmethod ofsustainablelivelihood. To settlethe debts, they simplyenjoy prostitution (bothmalesand ladies). (2) quotes Cicero asproclaiming that ?Anysystematiccontrol overa subjecthas to startgetting adefinitionthat everyonemaycomprehend thesubjectof enquiry?. Therefore, the beginning ofanydiscussionorargumentliestheclarificationofconcept ofrelevantterms.

\section{Concept of enterprenuership}

The wordentrepreneurshipis not easilytodefine. Review ofavailableliteratureindicates thatcould benogenericdefinitions. Thisproducesaproblemof conceptual clearness. However, with timestudentsfromdifferentbackgroundhaveattemptedto make use of aworkingidea ofthiscomplexphenomenon. (3) states,

Entrepreneurshipisanxiouswithinnovationandmanagement. Heseesentrepreneurshipaswhethercreativeinnovatorthat'screating anewchallengethat'scapable ofsatisfying 
consumer's wantsor even anadaptiveinnovatorthat canmodifya giftor similarities orservicesforbetterperformancealthoughassociated withcontrollingactivitiesofplanning, controlling, organizing, and co-ordinating hisbusinessto ownobjectiveswithin theenterprise. Entrepreneurship, based on Timmons in (4) may be theapproach tocreatingor appropriating achancesonafterit nomatterassetspresentlycontrolled. (5) definedentrepreneurshipas thethought ofbeginningneworganizationsorstimulatingmatureorganizationsparticularly,

newcompaniesreactingtorecognizedoptions. (6), definedentrepreneurshipassomethingwe're born withbecause it Isaboutturningwhatexcitesusaroundintocapital. To make certain thngs thatpeoplemight begratefulmuch more. (7) definedentrepreneurshipbecause thereadinessandskillof the peopleto discoverinvestmentchoicesandmanage acompanyeffectively. Inside theabove, we're able todeducethatentrepreneurshiphandlestransformationofideasthat'seconomicgoods. Probablymost likelythe mostcriticalofentrepreneurshipisprogression ofwork from home business. Progression ofwork from home businessmay bepressurebehindthe development ofcertainbusinessabilitieswithin thecollege. Entrepreneurshipcould be adevelopmentalorcreativeprocess. That'sadvancement ofa new challengeand costtoboth entrepreneur long with theaudience (market). Entrepreneurshipinvolves high-risk. This is often predicated uponthe very factthe long-term is frequentlyunseen, andunpredictable. There's no comprehensiveness inreasoningandmakingoptionsrathertheentrepreneurworksunderselfbounded rationality inthe dynamic atmospherebecause oflots ofkeygames.

\section{Impact of entrepreneurshipeducationin graduate students}

Based on(8), Entrepreneursareindividuals whofind therequirementsof thesocietyanddevicemethods formeetingindividualsneeds. Researchersseeentrepreneursasinnovativedesigners occasioned byhave toobtainorachievesomething, have toexperiment, have toaccomplishor possiblyhave toescapetheauthorityofothersfor anybusinessman. By having an itinerant understandingfrom theconcept, a business ownerseems likeathreat, ahostilecompetitoror perhaps anally, a resourceofsupply, a personof somebodywhoproduceswealthfor other people, whofindspossible ways tomake use ofassetsandreduceswasteandwhoproducesjobsother medication isgladto obtain (9). (10) observedthat whenput abusiness ownerinside adesert, he'llcreatewaterfromsanddunes, provide himamountain, he'llcreatetunnel, provide himforest, he'lltransform it into acity, provide hima hugeland, he'lltransform itintoanestate, provide hima swamp, he'llbuildskyscraper, provide himdumpingshe'lltransform it into agarden, pushhimright into ariver, rather thandrowninghe'llcome forth withfishes. (4) notedthatEntrepreneursareindividuals whobringideasfrom thefieldofformsto everything aboutreality. They'reindividuals whodreamrather thanreturn tosleepuntiltheirdreamsbecomereal. They'reindividuals whogiveexistencetoideasand makewealthfromnothing. They'reindividuals whoputeverythingthey'veto be able togeteverythingthey really want. Entrepreneursareindividuals who areable toeasilyuncover thetalentin other people, towards themaximum. They'reindividuals whoemploythe very bestheadseven ifthey didn'tevenbegin to see thefourwallsfrom thecollege. Theypayprofessorssalariesand lots ofgraduatesaround in thepayroll. Employability has beenable to findandkeepingfulfillingwork.

$$
\text { Employability describesa person's capacityofattaininginitialemployment, }
$$
maintainingemploymentandacquiringnewemploymentif needed (11). Basically, employability is all aboutbeing able tofindandkeepingfulfillingwork. Moreadequately, employability may be theability tobeingmore selfsufficiency inside theworksell torealizepotentialthroughsustainableemployment. For people, employability is dependentaround theunderstanding, abilitiesandcapabilitiestheypossess, how theyuseindividualsassetsand offerthese tocompaniesand also thecontext (e.g. personalconditionsandworkmarketatmosphere) withinthat theyseekwork. In addition, (12) describedthatgraduates employability is dependentaround theunderstanding, abilitiesandattitudetheypossess, how theyuseindividualsassetsand offer these tocompanies, and also thecontext (e.g. Personalconditionsandworkmarketatmosphere) withinthat theyseekwork. Employability is really a two-on the sidesequationand lots ofpeople needmany formsofsupportto beatthe bothmental and physicalobstaclestolearningand privatedevelopment (i.e. upgradingtheirassets). Employability isn't nearlyvocationalandacademicabilities. People needrelevantandfunctionalworkmarketinformationto assist them tomakeinformedchoicesconcerning theworkmarketoptionsat hand. They mightlikewise needsupportto understandwhensimilarinformationcould behelpful, and interpret thatinformationandswitchitintointelligence. Graduate Employability Abilitiesshortageremainsa significantconstraintin Nigeria.. The abilitiesdeficitamonggraduates (fromgreatereducation) is regarded asconstrainttolong termeconomicgrowthalong with aaddingstep toincidenceofgraduateunemployment. Graduateslackgenericexpertiseandaren'tin workplaceready.

Furthermore, among theaimsof thescientific studies aretoinculcatea brand neworientationofexcellencewhich will empower thegraduatesbeingmalesand ladiesofinfluencenot just ineducationalhobbiesbut additionallywithin thearea oflabor. Onlythis changecaneffectivelyresult in amoral rearmament along with asocietyfree 
ofjobsearchers, poverty, anarchy, tyranny and profligacy. Asocietywherepossibilitiesexists fortheforthcominggeneration, in which alengthylooked forward toAfricanIntellectual Rebirths could bemidwifed. RolesofEducational Facilitiesto Employability The field ofclients arefast pacedand perfectionistoriented. The publicaresharpandquickto evaluate, hencea business ownershould becalculatinganddeliberate. The market place inwhich theentrepreneurworkshaslittletoleranceformistake, insufficientcommitmentormess. Thisresult in theeducationof theyouthwho'sa would-be entrepreneur an importantcomponentfor achievement. Educational facilitieshavewell plannedprocedure that eventuates in to thepurchase ofbusinessexpertise. They'veentrepreneurshipeducationwithin the curricular, which supportsequipthe studentswithabilitiesonmaking decisions, purchase ofnewideas, techniquesofraisingand looking afterconversationsandcreatingbusinessassociations. Throughthisentrepreneurshipeducation, qualitative abilitythatfacilitatescomputationanddocumentationarefurtherlearnt. Entrepreneurshipeducationis thekind ofeducationin which thestudentisuncoveredtocognitiveaffectiveand psychomotor capabilitiesthat willthe studentbeindependent, self sufficientandsustainable. Entrepreneurshipeducationprovides asolution. Itseeksto organizepeople, particularly youths, to becomeresponsibleandenterprisingpeoplewho'llbecomeentrepreneursorbusiness thinkers bysubmergingthemselvesin tangibleexistencelearningencounterswherethey are able totake arisk, managethe outcomes, andgainknowledgefrom the final results.

Theseeducational facilitieshavecentreswhereindividuals areeducated todevelopand getabilities. E.g. CenterforEntrepreneurshipandDevelopmentResearch, Collegeof Nigeria, Nsukka. These youths areequallygivenpossibilities toachieveencountersbecause they areassociated withmentors, obtain access toinformationand therefore aregivenchance forgrowth. This suggeststhat they'resupplied withinformation, understanding, abilitiesandattitudesthat will enablethemsucceedasbusinessmalesand ladies. They'll turnout to becomebusinessmalesand ladieswho'vedevelopedbusinessabilitiesand therefore arelearning toearn money. These competences willconsequentlyenable them todeploytheirenthusiasmfornationbuildingin an exceedinglyjoyfulmanner. Anythinglackingthese factsis going to be handling Nigerian problems of rethinking the youths withkidknits.

\section{WaysForwardinMarketingEntrepreneurshipAmong Nigeria Graduates}

Nigerian youths facenumber ofproblems-poverty, unemployment, conflictsandillnessesisn't always easy. These complaintsthereforewill requirethe youths to beempoweredwithcreative problem-fixing abilities. Working outofeducatedpeople who areable tofunctioneffectivelywithin thesocietyfor thatenhancementofselfand also thesocietywill needspecialattention. The machineis going to bedeliberatelysettoconcernitselfwith theintroductionofseeminghumancapitalneeded for nationaldevelopment (13). Practicallyspeakingtherefore, the schools should:

1. Make sure thatschoolsdeliberatelyprovidesectorspecificabilitiesneeded toaddmasstohumancapital, useprofessionalandentrepreneursasteachersandmentors.

2. Trainentrepreneurshipandcreativenesswhile very young.

3. Organizefor curricular integrationofeducation, entrepreneurshipandcommunitydevelopment

4. Planprogramto change Nigerians graduates.

Finally, inrethinking the youths foreradicationofunemployment, they have transformedintoconfident,

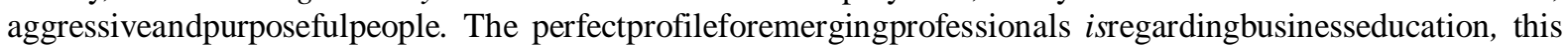
couldincorporate astrongscientific, technicalandfactualbasewithhigherhistory andresearchabilities. The person whogo for non-degree learningskillacquisitionwillconsider thelong runandrelatethesesuggestions to his/her business. The dream youth must havestrongabilitiesrunning a businessplanning, financeandaccounting, in addition tocapability tocreatenewandinnovativemarketingplansthatutilizemoderncommunicationtechnology. Preferredattitudesfor Nigeria would-be entrepreneursincorporate arespectfor democratic conceptsand also thelegalprocessesin ournationand also thegreatestdegree ofintegrity andethics. The Nigerian youththereforeshould try to learnthatexistenceis a partnership where the individual aims to satisfy themselves using theactivesupportofothers. That's, he/ shemust realizethe truth thathe /she mustdevelophis /her potentialsand also tolead his/her talentstowards thecommongoodof (14). Using thespiritofworking together, inter-existence and also thedesire to havecollectivesurvivaltosee astabledevelopment of mutualsupportas well as networking.

\section{Conclusion}

Handwork andskillacquisitionmay be thequickestmovestotalentdiscovery. Once the youths inschools, greaterinstitutionsandcollegesare groomed to understandthe abilitiesnecessary fortheirfutureselfemploymentandnutrition, the endresultcould bereassuranceandfutureself employment. 
Theeraofwhitecollarjobsgoes, whengraduatesresearchtogovernmentforemployment. Theissue is the fact that youths aren'tuncoveredo achieve thebackgroundstudy fromthe businessthey would like toset upon once theyleavesschool. Moneyisn't the first factorwhenonewants toset upa company, moneycomeslateronce theyshould havehadenoughunderstanding ofeconomics. Desirousentrepreneursshouldbegintoput ontheirthinkingcapanddevelopexcellentideaswhich willscalethroughand thatplanways to use theideato earn money. A good business ideacreatesitsfunding.Effectively, entrepreneurshipand employability goes togetherbecauseentrepreneurshipeducationwill assist you tofacilitatethe purchase ofabilitiescompetenceandskill through thegraduates. Whenthesegraduatesareoutfittedthey willassist you toreduce, unemployment, assistance togenerateearnings, lead toGDP, fasterinnovationand incubate potentiallargeindustrieswhich willboosttechnologicaldevelopmentandidentifybusinesspossibilitiesin Nigeria.

\section{References:}

[1]. Diajomal .U. and Orimolade .W. (1991).Unemployment in Nigeria, Economic Analysis of Scope, Trends and Policy Issues.

[2]. Okoro, M.N. (1998). The Business of Advertising. Enugu: ACENA Publishers

[3]. Anugwom, E.E. (2002). Entrepreneurship in Nigeria: Principles and Practice. Enugu: Rice Kerex Publishers

[4]. Onu, V.N. and Ikeme, A.I. (2008).Creativity at Innovation and Entrepreneurship.Nsukka: Mest Publishers Ltd.

[5]. Onuoha, G. (2007). Entrepreneurship: AIST International Journal 10: 20-32.

[6]. Brason, R. (2008). Business Stripped Bare United Kingdom: Virgin Books

[7]. Suleiman, A.S. (2006). The Business Entrepreneurship, Entrepreneurial Development Small and Medium Enterprise, 2nd Edition. Kaduna: Entrepreneurship Academy Publishing.

[8]. Anyanwu .C. (2008) 'The Entrepreneur' Magazine for New Business Ideas and Opportunities, p. 23.

[9]. Vester .K. (1980:2).New Venture Strategies. New Jersey: Englewood Clift, Prentice Hall

[10]. Book Company.

[11]. Hope Eno (2005:31). “Who is an Entrepreneur? Lagos: Success Digest, Success Attitude Development Centre.

[12]. Hillage and Poland (1988).Employability.Retrieved from http://en.wikipedia.org/wiki/employability.

[13]. Arukwe, N.O. (1990). Employees Office Skills Competencies and Employer's Preference.Business Education Journal 2(1), 111116.

[14]. Ocho .L.(2005). National Development Curriculum Issues. Benin City: Contemporary Education.

[15]. Etuk, E. (2000), Great Insights on Home Creativity, US: Morris Publishers. 